MATHEMATICS OF COMPUTATION

Volume 66, Number 220, October 1997, Pages 1441-1459

S $0025-5718(97) 00905-8$

\title{
CONTINUOUS COLLOCATION APPROXIMATIONS TO SOLUTIONS OF FIRST KIND VOLTERRA EQUATIONS
}

\author{
J.-P. KAUTHEN AND H. BRUNNER
}

\begin{abstract}
In this paper we give necessary and sufficient conditions for convergence of continuous collocation approximations of solutions of first kind Volterra integral equations. The results close some longstanding gaps in the theory of polynomial spline collocation methods for such equations. The convergence analysis is based on a Runge-Kutta or ODE approach.
\end{abstract}

\section{INTRODUCTION}

In this paper we consider first kind Volterra integral equations of the form

$$
\int_{0}^{t} K(t, s) y(s) d s=g(t), \quad t \in I=[0, T] .
$$

Here $g$ and $K$ are supposed to be sufficiently smooth functions satisfying $g(0)=0$ and $|K(t, t)| \geq \kappa>0$ for all $t \in I$.

The (unique) solution of (1.1) is to be approximated in certain polynomial spline spaces. In the case where this space is $S_{m}^{(-1)}\left(\Pi_{N}\right)$, the space of discontinuous polynomial spline functions of degree $m$, the relationship between the choice of the collocation parameters and the (order of) convergence of the collocation solution is well understood (compare [2], [4]). The picture is much more complex for the space $S_{m}^{(0)}\left(\Pi_{N}\right)$ of continuous polynomial splines, especially when the set of collocation points does not contain the mesh points. It is the purpose of this paper to develop a convergence theory analogous to the one for $S_{m}^{(-1)}\left(\Pi_{N}\right)$. It has been shown in [12] that methods based on splines with full continuity and of degree greater than one are divergent.

We also discuss the corresponding fully discretized collocation methods. These methods were introduced in [10], [11], and their place within the framework of polynomial spline collocation methods was described in [1], [2] (compare also [4]).

In Section 2, we define the continuous collocation approximations in $S_{m}^{(0)}\left(\Pi_{N}\right)$. Section 3 contains some preliminary and technical results concerning properties of the coefficients of the implicit Runge-Kutta method defined by the collocation parameters. The main convergence results are presented in Section 4 where we give proofs for equations with constant kernel. The convergence analysis for equations with non-constant kernel is carried out in Section 5. In Section 6 we briefly discuss

Received by the editor March 16, 1995.

1991 Mathematics Subject Classification. Primary 65R20, 45L10.

Key words and phrases. Integral equation, collocation method, Runge-Kutta method.

(C) 1997 American Mathematical Society 
the fully discretized collocation approximations and give a numerical illustration. Finally Section 7 contains some concluding remarks.

\section{Continuous collocation approximations}

Consider the subdivision $\Pi_{N}$ of the interval $I=[0, T]$ defined by $t_{n}=n h$, $n=0, \ldots, N$, where the (constant) stepsize is given by $h=T / N(N>0)$. Let $0<$ $c_{1}<c_{2}<\ldots<c_{m} \leq 1(m \geq 1)$ be the collocation parameters and $t_{n j}=t_{n}+c_{j} h$, $j=1, \ldots, m, n=0, \ldots, N-1$, the collocation points.

We seek an approximation $u$ in the spline space $S_{m}^{(0)}\left(\Pi_{N}\right)$ of the solution $y$ of (1.1). This approximation satisfies the collocation equation

$$
\int_{0}^{t_{n j}} K\left(t_{n j}, s\right) u(s) d s=g\left(t_{n j}\right), \quad j=1, \ldots, m, n=0, \ldots, N-1,
$$

and the continuity conditions

$$
u_{n-1}\left(t_{n}\right)=u_{n}\left(t_{n}\right), \quad n=1, \ldots, N-1 .
$$

Here $u_{n}$ denotes the restriction of $u$ to the subinterval $\left[t_{n}, t_{n+1}\right]$. The initial value is $u(0)=y(0)=g^{\prime}(0) / K(0,0)$.

On each subinterval $\left[t_{n}, t_{n+1}\right], n=0, \ldots, N-1$, the approximation $u$ is a polynomial of degree $m$ and is represented by the interpolation formula

$$
u_{n}\left(t_{n}+\tau h\right)=L_{0}(\tau) u_{n-1}\left(t_{n}\right)+\sum_{\ell=1}^{m} L_{\ell}(\tau) u_{n}\left(t_{n \ell}\right), \quad \tau \in[0,1],
$$

where the Lagrange polynomials $L_{\ell}, \ell=0, \ldots, m$, are defined by

$$
L_{0}(\tau)=(-1)^{m} \prod_{k=1}^{m} \frac{\tau-c_{k}}{c_{k}}, \quad L_{\ell}(\tau)=\frac{\tau}{c_{\ell}} \prod_{\substack{k=1 \\ k \neq \text { ell }}}^{m} \frac{\tau-c_{k}}{c_{\ell}-c_{k}}, \quad \ell=1, \ldots, m .
$$

The collocation approximation $u$ is obtained by solving on each subinterval $\left[t_{n}, t_{n+1}\right], n=0, \ldots, N-1$, the following system:

$$
\begin{aligned}
h \sum_{\ell=1}^{m}\left(\int_{0}^{c_{j}} K\left(t_{n j}, t_{n}+\tau h\right) L_{\ell}(\tau) d \tau\right) u_{n}\left(t_{n \ell}\right) \\
=g\left(t_{n j}\right)-h\left(\int_{0}^{c_{j}} K\left(t_{n j}, t_{n}+\tau h\right) L_{0}(\tau) d \tau\right) u_{n-1}\left(t_{n}\right) \\
-h \sum_{\nu=0}^{n-1}\left(\left(\int_{0}^{1} K\left(t_{n j}, t_{\nu}+\tau h\right) L_{0}(\tau) d \tau\right) u_{\nu-1}\left(t_{\nu}\right)\right. \\
\left.\quad+\sum_{\ell=1}^{m}\left(\int_{0}^{1} K\left(t_{n j}, t_{\nu}+\tau h\right) L_{\ell}(\tau) d \tau\right) u_{\nu}\left(t_{\nu \ell}\right)\right)
\end{aligned}
$$

$(j=1, \ldots, m)$, with $u_{-1}\left(t_{0}\right)=u_{0}(0)$. This system has a unique solution if $h$ is sufficiently small and if the matrix $\left(\int_{0}^{c_{j}} L_{\ell}(\tau) d \tau\right)_{j, \ell=1}^{m}$ is invertible (see Section 3). Having solved this system, one obtains the approximation at $t_{n+1}$ by

$$
u_{n}\left(t_{n+1}\right)=L_{0}(1) u_{n-1}\left(t_{n}\right)+\sum_{\ell=1}^{m} L_{\ell}(1) u_{n}\left(t_{n \ell}\right) .
$$




\section{Preliminary Considerations}

We define $c_{0}=0$ and consider the $m+1$-stage implicit Runge-Kutta method which is equivalent to the collocation method characterized by $c_{i}, i=0, \ldots, m$. The coefficients of this method are then given by

$$
a_{j \ell}=\int_{0}^{c_{j}} L_{\ell}(\tau) d \tau, \quad b_{\ell}=\int_{0}^{1} L_{\ell}(\tau) d \tau, \quad j, \ell=1, \ldots, m .
$$

It follows from Theorem II.7.8 of [8] that the matrix $A=\left(a_{j \ell}\right)_{j, \ell=0}^{m}$ is defined by condition $C(m+1)$. The vector $b=\left(b_{0}, \ldots, b_{m}\right)^{\top}$ is defined by condition $B(m+1)$. We recall that an $s$-stage Runge-Kutta method satisfies

$$
\begin{array}{ll}
B(p) \quad \text { if } \quad \sum_{i=1}^{s} b_{i} c_{i}^{k-1}=\frac{1}{k}, \quad k=1, \ldots, p, \\
C(q) \quad \text { if } \quad \sum_{j=1}^{s} a_{i j} c_{j}^{k-1}=\frac{c_{i}^{k}}{k}, \quad i=1, \ldots, s, \quad k=1, \ldots, q,
\end{array}
$$

(see e.g. [8, p. 208]). Hence the coefficients $a_{i j}$ and $b_{i}$ satisfy

$$
\begin{gathered}
\sum_{j=0}^{m} a_{i j} c_{j}^{q-1}=\frac{c_{i}^{q}}{q}, \quad i=0, \ldots, m, \quad q=1, \ldots, m+1, \\
\sum_{i=0}^{m} b_{i} c_{i}^{q-1}=\frac{1}{q}, \quad q=1, \ldots, m+1 .
\end{gathered}
$$

It follows that $a_{0 \ell}=0, \ell=0, \ldots, m$. We define $\widehat{A}=\left(a_{j \ell}\right)_{j, \ell=1}^{m}, a_{0}=\left(a_{10}, \ldots, a_{m 0}\right)^{\top}$, $\hat{c}=\left(c_{1}, \ldots, c_{m}\right)^{\top}$ and $\hat{b}=\left(b_{1}, \ldots, b_{m}\right)^{\top}$. Let $\mathbb{1}_{m}=(1, \ldots, 1)^{\top} \in \mathbb{R}^{m}$ and $R(z)=$ $1+z b^{\top}(I-z A)^{-1} \mathbb{1}_{m+1}$ denote the stability function of the Runge-Kutta method (see e.g. [9, pp. 40]). Some properties of the coefficients of the Runge-Kutta method are stated in the following lemma.

Lemma 1. Let $A=\left(a_{j \ell}\right)_{j, \ell=0}^{m}, b=\left(b_{0}, \ldots, b_{m}\right)^{\top}$ and $c=\left(c_{0}, \ldots, c_{m}\right)^{\top}$ be the coefficients of the Runge-Kutta method defined above. Then

a) if $c_{m}<1, \lim _{z \rightarrow \infty} R(z)=\infty$ and if $c_{m}=1$,

$$
R(\infty)=(-1)^{m} \prod_{i=1}^{m-1} \frac{1-c_{i}}{c_{i}}
$$

b) the matrix $\widehat{A}=\left(a_{j \ell}\right)_{j, \ell=1}^{m}$ is invertible;

c) if $d=\left(d_{1}, \ldots, d_{m}\right)^{\top}$ is defined by $d=\widehat{A}^{-1} a_{0}$, we have

$$
d_{i}=(-1)^{m-1} \prod_{\substack{k=1 \\ k \neq i}}^{m} \frac{c_{i}-c_{k}}{c_{k}}, \quad i=1, \ldots, m ;
$$

d) it holds that

$$
1-\hat{b}^{\top} \widehat{A}^{-1} \mathbb{1}_{m}=L_{0}(1)\left(1+\sum_{i=1}^{m} \frac{1}{c_{i}}\right)
$$


e) if $\gamma=\left(\gamma_{1}, \ldots, \gamma_{m}\right)^{\top}$ is defined by $\gamma=\widehat{A}^{-1} \mathbb{1}_{m}$, we have

$$
\gamma_{i}=\left(\frac{1}{c_{i}}+\sum_{j=1}^{m} \frac{1}{c_{j}}\right) d_{i}, \quad i=1, \ldots, m
$$

f) it holds that

$$
\hat{b}^{\top} \widehat{A}^{-1} \hat{c}=1-L_{0}(1) .
$$

Proof. a) Let $M(\tau)=\frac{\tau}{(m+1) !} \prod_{i=1}^{m}\left(\tau-c_{i}\right)$. Then $M(0)=0$. If $c_{m}<1$, Theorem IV.3.9 of [9] implies that $\lim _{z \rightarrow \infty} R(z)=\lim _{z \rightarrow \infty} \frac{M(1)}{M^{\prime}(0)} z=\infty$. If $c_{m}=1, M(1)=0$ and it follows that $R(\infty)=\frac{M^{\prime}(1)}{M^{\prime}(0)}$ which gives (3.6).

b) $\widehat{A}$ is solution of

$$
\sum_{j=1}^{m} a_{i j} c_{j}^{q-1}=\frac{c_{i}^{q}}{q}, \quad i=1, \ldots, m, \quad q=2, \ldots, m+1,
$$

or

$$
\left(\begin{array}{ccc}
a_{11} & \ldots & a_{1 m} \\
\vdots & & \vdots \\
a_{m 1} & \ldots & a_{m m}
\end{array}\right)\left(\begin{array}{ccc}
c_{1} & \ldots & c_{1}^{m} \\
\vdots & & \vdots \\
c_{m} & \ldots & c_{m}^{m}
\end{array}\right)=\left(\begin{array}{ccc}
\frac{c_{1}^{2}}{2} & \ldots & \frac{c_{1}^{m+1}}{m+1} \\
\vdots & & \vdots \\
\frac{c_{m}^{2}}{2} & \ldots & \frac{c_{m}^{m+1}}{m+1}
\end{array}\right) .
$$

Since the $c_{i}$ are all different, all matrices are invertible.

c) We have

$$
\begin{gathered}
\left(\begin{array}{ccc}
a_{11} & \ldots & a_{1 m} \\
\vdots & & \vdots \\
a_{m 1} & \ldots & a_{m m}
\end{array}\right)\left(\begin{array}{ccc}
c_{1} & \ldots & c_{1}^{m} \\
\vdots & & \vdots \\
c_{m} & \ldots & c_{m}^{m}
\end{array}\right) \\
=\left(\begin{array}{ccc}
c_{1} & \ldots & c_{1}^{m} \\
\vdots & & \vdots \\
c_{m} & \ldots & c_{m}^{m}
\end{array}\right)\left(\begin{array}{ccccc}
0 & 0 & \ldots & 0 & \frac{-\beta_{0}}{m+1} \\
\frac{1}{2} & 0 & \ldots & 0 & \frac{-\beta_{1}}{m+1} \\
0 & \frac{1}{3} & \ldots & 0 & \frac{-\beta_{2}}{m+1} \\
\vdots & \vdots & \ddots & \vdots & \vdots \\
0 & 0 & \ldots & \frac{1}{m} & \frac{-\beta_{m-1}}{m+1}
\end{array}\right),
\end{gathered}
$$

or in matrix notation

$$
\widehat{A} V=V \Omega \text {. }
$$

Here $\beta_{i}, i=0, \ldots, m$, are the coefficients of the polynomial

$$
M(t)=\prod_{i=1}^{m}\left(t-c_{i}\right)=t^{m}+\beta_{m-1} t^{m-1}+\ldots+\beta_{1} t+\beta_{0} .
$$

Relation (3.4) implies that $a_{0}=\hat{c}-\widehat{A} \mathbb{1}_{m}$. Therefore $d=x-\mathbb{1}_{m}$ where $x$ is the solution of $\widehat{A} x=c$. It follows from (3.11) that $V \Omega V^{-1} x=c$. Let $y=V^{-1} x$. Then $V \Omega y=c$ or $\Omega y=V^{-1} c=e_{1}$ with $e_{1}=(1,0, \ldots, 0)^{\top} \in \mathbb{R}^{m}$. We thus solve $\Omega y=e_{1}$, compute $x=V y$ and obtain $d=x-\mathbb{1}_{m}$. With $\beta_{m}=1$, we obtain $y_{j}=-(j+1) \frac{\beta_{j}}{\beta_{0}}, j=1, \ldots, m$. Hence

$$
x_{i}=\sum_{j=1}^{m} c_{i}^{j} y_{j}=-\sum_{j=1}^{m} c_{i}^{j}(j+1) \frac{\beta_{j}}{\beta_{0}} .
$$


Let $\widetilde{M}(t)=t M(t)$. Then

$$
\widetilde{M}^{\prime}\left(c_{i}\right)=c_{i} \prod_{\substack{k=1 \\ k \neq i}}^{m}\left(c_{i}-c_{k}\right)=\sum_{j=0}^{m}(j+1) \beta_{j} c_{i}^{j} .
$$

This leads to

$$
x_{i}=-\frac{1}{\beta_{0}}\left(\sum_{j=0}^{m}(j+1) \beta_{j} c_{i}^{j}-\beta_{0}\right)=-\frac{1}{\beta_{0}} c_{i} \prod_{\substack{k=1 \\ k \neq i}}^{m}\left(c_{i}-c_{k}\right)+1
$$

and

$$
d_{i}=x_{i}-1=(-1)^{m-1} \prod_{\substack{k=1 \\ k \neq i}}^{m} \frac{c_{i}-c_{k}}{c_{k}}
$$

since $\beta_{0}=M(0)=(-1)^{m} \prod_{i=1}^{m} c_{i}$.

d) We have

$$
\widehat{A} V=\widetilde{V} J
$$

and

$$
\hat{b}^{\top} V=\mathbb{1}_{m}^{\top} J
$$

where

$$
\widetilde{V}=\left(\begin{array}{ccc}
c_{1} & \ldots & c_{1}^{m+1} \\
\vdots & & \vdots \\
c_{m} & \ldots & c_{m}^{m+1}
\end{array}\right), \quad J=\left(\begin{array}{cccc}
0 & 0 & \ldots & 0 \\
\frac{1}{2} & 0 & \ldots & 0 \\
0 & \frac{1}{3} & \ldots & 0 \\
\vdots & \vdots & \ddots & \vdots \\
0 & 0 & \ldots & \frac{1}{m+1}
\end{array}\right) .
$$

We solve (3.12) for $V$ and insert into (3.13). Thus

$$
\hat{b}^{\top} \widehat{A}^{-1} \widetilde{V} J=\mathbb{1}_{m}^{\top} J
$$

We now have to find $u=\left(u_{1}, \ldots, u_{m}\right)^{\top} \in \mathbb{R}^{m}$ such that

$$
\widetilde{V} J u=\mathbb{1}_{m} .
$$

It then follows that $\hat{b}^{\top} \widehat{A}^{-1} \mathbb{1}_{m}=\hat{b}^{\top} \widehat{A}^{-1} \widetilde{V} J u=\mathbb{1}_{m}^{\top} J u$ or $1-\hat{b}^{\top} \widehat{A}^{-1} \mathbb{1}_{m}=1-\mathbb{1}_{m}^{\top} J u$. Condition (3.14) is equivalent to

$$
\sum_{k=1}^{m} u_{k} \frac{c_{i}^{k+1}}{k+1}=1, \quad i=1, \ldots, m
$$

Let the polynomial $p \in \pi_{m}$ be defined by $p(t)=\sum_{k=1}^{m} u_{k} t^{k}$. Then $\int_{0}^{c_{i}} p(t) d t=$ $\sum_{k=1}^{m} u_{k} \frac{c_{i}^{k+1}}{k+1}$. By (3.15) we thus seek $p$ such that $\int_{0}^{c_{i}} p(t) d t=1, i=1, \ldots, m$. Let $q(t)=\int_{0}^{t} p(s) d s$. Then $q \in \pi_{m+1}, q^{\prime}(t)=p(t)$ and $q$ is the solution of the interpolation problem

$$
q\left(c_{i}\right)=1, \quad i=1, \ldots, m, \quad q(0)=0, \quad q^{\prime}(0)=0 .
$$

One easily verifies that

$$
q(t)=1+(\alpha+\beta t) \prod_{i=1}^{m}\left(t-c_{i}\right), \quad \alpha=\frac{(-1)^{m-1}}{\prod_{i=1}^{m} c_{i}}, \quad \beta=\alpha \sum_{i=1}^{m} \frac{1}{c_{i}} .
$$


We now have

$$
\mathbb{1}_{m}^{\top} J u=\sum_{k=1}^{m} \frac{u_{k}}{k+1}=q(1)
$$

and find that

$$
1-\hat{b} \widehat{A}^{-1} \mathbb{1}_{m}=1-\mathbb{1}_{m}^{\top} J u=1-q(1)=(-1)^{m}\left(\prod_{i=1}^{m} \frac{1-c_{i}}{c_{i}}\right)\left(1+\sum_{i=1}^{m} \frac{1}{c_{i}}\right) .
$$

e) We have $\gamma=V u$ where $\widetilde{V} J u=\mathbb{1}_{m}$ (see (3.12) and (3.14)). Thus $\gamma_{i}=$ $\sum_{j=1}^{m} c_{i}^{j} u_{j}=p\left(c_{i}\right)=q^{\prime}\left(c_{i}\right)$ where $p$ and $q$ are as in part d) and the desired result is readily obtained.

f) The proof is similar to that of part d). One has $\hat{b}^{\top} \widehat{A}^{-1} \hat{c}=q(1)$ where $q$ is the solution of the interpolation problem

$$
q\left(c_{i}\right)=c_{i}, \quad i=1, \ldots, m, \quad q(0)=0, \quad q^{\prime}(0)=0 .
$$

This completes the proof of the lemma.

\section{Convergence analysis}

We now return to the collocation method. The exact solution of (1.1) can be written on $\left[t_{n}, t_{n+1}\right]$ as

$$
y\left(t_{n}+\tau h\right)=L_{0}(\tau) y\left(t_{n}\right)+\sum_{\ell=1}^{m} L_{\ell}(\tau) y\left(t_{n \ell}\right)+r_{n}(\tau), \quad \tau \in[0,1],
$$

where the interpolation error is

$$
r_{n}(\tau)=h^{m+1} \frac{y^{(m+1)}\left(\xi_{n}(\tau)\right)}{(m+1) !} \tau \prod_{i=1}^{m}\left(\tau-c_{i}\right), \quad t_{n}<\xi_{n}(\tau)<t_{n+1} .
$$

It follows from (4.1) and (2.3) that the collocation error $e=y-u$ is given by

$$
e_{n}\left(t_{n}+\tau h\right)=L_{0}(\tau) e_{n-1}\left(t_{n}\right)+\sum_{\ell=1}^{m} L_{\ell}(\tau) e_{n}\left(t_{n \ell}\right)+r_{n}(\tau)
$$

We have in particular that $e_{n}\left(t_{n}\right)=e_{n-1}\left(t_{n}\right)$, with $e_{n}$ denoting the restriction of $e$ to $\left[t_{n}, t_{n+1}\right]$. Subtracting the collocation equation (2.1) from (1.1), we obtain the error recursion

$$
\begin{array}{rl}
\int_{0}^{c_{j}} & K\left(t_{n j}, t_{n}+\tau h\right) e_{n}\left(t_{n}+\tau h\right) d \tau \\
& =-\sum_{\nu=0}^{n-1} \int_{0}^{1} K\left(t_{n j}, t_{\nu}+\tau h\right) e_{\nu}\left(t_{\nu}+\tau h\right) d \tau, \quad j=1, \ldots, m .
\end{array}
$$

We first consider the case of constant kernel $K(t, s) \equiv 1$. This case already contains all important ideas. The convergence analysis for equations with non-constant 
kernels is carried out in Section 5. We rewrite (4.4) with $n$ replaced by $n-1$ and with $j=m$ and subtract it from (4.4). We obtain

$$
\begin{aligned}
& \int_{0}^{c_{j}} e_{n}\left(t_{n}+\tau h\right) d \tau \\
& \quad=\int_{0}^{c_{m}} e_{n-1}\left(t_{n-1}+\tau h\right) d \tau-\int_{0}^{1} e_{n-1}\left(t_{n-1}+\tau h\right) d \tau, \quad j=1, \ldots, m .
\end{aligned}
$$

We have to distinguish between two cases: $c_{m}=1$ and $c_{m}<1$.

Case I: $c_{m}=1$. This case has already been studied in [2]. The techniques employed here are different and rely on an "ODE approach". Equation (4.5) now reduces to

$$
\int_{0}^{c_{j}} e_{n}\left(t_{n}+\tau h\right) d \tau=0, \quad j=1, \ldots, m .
$$

Inserting (4.3) into (4.6), we obtain

$$
a_{j 0} e_{n-1}\left(t_{n}\right)+\sum_{\ell=1}^{m} a_{j \ell} e_{n}\left(t_{n \ell}\right)+\int_{0}^{c_{j}} r_{n}(\tau) d \tau=0, \quad j=1, \ldots, m .
$$

Since, with $c_{m}=1, e_{n-1}\left(t_{n-1 m}\right)=e_{n-1}\left(t_{n}\right)$, the last equation becomes

$$
\sum_{\ell=1}^{m} a_{j \ell} e_{n}\left(t_{n \ell}\right)=-a_{j 0} e_{n-1}\left(t_{n-1 m}\right)+r_{n j}, \quad j=1, \ldots, m,
$$

where $r_{n j}=-\int_{0}^{c_{j}} r_{n}(\tau) d \tau$. In matrix notation

$$
\widehat{A} E_{n}=B E_{n-1}+R_{n}
$$

or

$$
E_{n}=\widehat{A}^{-1} B E_{n-1}+\widehat{A}^{-1} R_{n}
$$

where $E_{n}=\left(e_{n}\left(t_{n 1}\right), \ldots, e_{n}\left(t_{n m}\right)\right)^{\top}, \widehat{A}=\left(a_{j \ell}\right)_{j, \ell=1}^{m}, R_{n}=\left(r_{n 1}, \ldots, r_{n m}\right)^{\top}$ and

$$
B=\left(\begin{array}{cccc}
0 & \ldots & 0 & -a_{10} \\
\vdots & & \vdots & \vdots \\
0 & \ldots & 0 & -a_{m 0}
\end{array}\right) .
$$

Part c) of Lemma 1 implies that

$$
\widehat{A}^{-1} B=\left(\begin{array}{cccc}
0 & \ldots & 0 & -d_{1} \\
\vdots & & \vdots & \vdots \\
0 & \ldots & 0 & -d_{m}
\end{array}\right) .
$$

The only non-zero eigenvalue of this matrix is $-d_{m}$. Note that

$$
-d_{m}=R(\infty)=(-1)^{m} \prod_{i=1}^{m-1} \frac{1-c_{i}}{c_{i}},
$$

(see parts a) and c) of Lemma 1). It is easy to show that

$$
\left(\widehat{A}^{-1} B\right)^{n}=(-1)^{n-1} d_{m}^{n-1} \widehat{A}^{-1} B .
$$


It follows from (4.7) that

$$
E_{n}=\left(\widehat{A}^{-1} B\right)^{n} E_{0}+\sum_{\nu=1}^{n}\left(\widehat{A}^{-1} B\right)^{n-\nu} \widehat{A}^{-1} R_{\nu} .
$$

With (4.9) this equation becomes

$$
E_{n}=(-1)^{n-1} d_{m}^{n-1} \widehat{A}^{-1} B E_{0}+\sum_{\nu=1}^{n-1}(-1)^{n-\nu-1} d_{m}^{n-\nu-1} \widehat{A}^{-1} B \widehat{A}^{-1} R_{\nu}+\widehat{A}^{-1} R_{n} .
$$

If $\left|d_{m}\right|<1$, there exist constants $D_{1}$ and $D_{2}$ such that for $n=1, \ldots, N-1$,

$$
\left\|E_{n}\right\| \leq D_{1}\left\|E_{0}\right\|+D_{2} \max _{\nu=1, \ldots, n}\left\|R_{\nu}\right\| .
$$

With $e(0)=0$ it is easy to show that $\left\|E_{0}\right\|=\mathcal{O}\left(h^{m+1}\right)$. Moreover $\left\|R_{n}\right\|=\mathcal{O}\left(h^{m+1}\right)$, $n=1, \ldots, N(\operatorname{see}(4.2))$. Finally there exists a constant $C$ such that

$$
\left\|E_{n}\right\| \leq C h^{m+1}, \quad n=1, \ldots, N-1 .
$$

From (4.3) we then obtain that

$$
\|e\|_{\infty}=\sup _{t \in I}|e(t)|=\mathcal{O}\left(h^{m+1}\right), \quad h \rightarrow 0, N h=T .
$$

If $d_{m}=1$ (i.e. $R(\infty)=-1$ and $m$ is odd), (4.10) becomes

$$
\begin{aligned}
E_{n}=( & -1)^{n-1} \widehat{A}^{-1} B E_{0}+\widehat{A}^{-1} B \widehat{A}^{-1}\left(\left(R_{n-1}-R_{n-2}\right)+\left(R_{n-3}-R_{n-4}\right)+\ldots \pm R_{1}\right) \\
& +\widehat{A}^{-1} R_{n} .
\end{aligned}
$$

Since $R_{j}-R_{j-1}=\mathcal{O}\left(h^{m+2}\right)$ if $y \in C^{m+2}(I)$ (see (4.2)), it follows that

$$
\left\|E_{n}\right\| \leq C h^{m+1}, \quad n=1, \ldots, N-1 .
$$

If $d_{m}=-1$ (i.e. $R(\infty)=1$ and $m$ is even), (4.10) becomes

$$
E_{n}=\widehat{A}^{-1} B E_{0}+\sum_{\nu=1}^{n-1} \widehat{A}^{-1} B \widehat{A}^{-1} R_{\nu}+\widehat{A}^{-1} R_{n}
$$

and the method converges only with order $m$, i.e.

$$
\left\|E_{n}\right\| \leq C h^{m}, \quad n=1, \ldots, N-1 .
$$

If $\left|d_{m}\right|>1$, the method diverges. For the convergence analysis in the case of a general, non-constant kernel, we refer to Section 5. We have the following theorem, based on our "ODE approach" (compare with Theorem 5.5.1b of [4]).

Theorem 1. Assume that $g$ and $K$ in (1.1) are of class $C^{m+3}$. Let $u$ be the collocation approximation in $S_{m}^{(0)}\left(\Pi_{N}\right)$ of the solution $y$ of (1.1) and defined by (2.3)-(2.6). If $c_{m}=1$, then the collocation approximation u converges to the solution $y$ for any $m \geq 1$ if and only if

$$
-1 \leq R(\infty)=(-1)^{m} \prod_{i=1}^{m-1} \frac{1-c_{i}}{c_{i}} \leq 1,
$$

and the collocation error $e=y-u$ satisfies

$$
\|e\|_{\infty}=\sup _{t \in I}|e(t)|=\left\{\begin{array}{ll}
\mathcal{O}\left(h^{m+1}\right) & \text { if }-1 \leq R(\infty)<1, \\
\mathcal{O}\left(h^{m}\right) & \text { if } R(\infty)=1,
\end{array} \rightarrow 0, N h=T .\right.
$$


Case II: $c_{m}<1$. Inserting (4.3) into (4.5) we obtain for $j=1, \ldots, m$,

$$
\begin{aligned}
a_{j 0} e_{n-1}\left(t_{n}\right)+\sum_{\ell=1}^{m} a_{j \ell} e_{n}\left(t_{n \ell}\right)-r_{n j} \\
=a_{m 0} e_{n-2}\left(t_{n-1}\right)+\sum_{\ell=1}^{m} a_{m \ell} e_{n-1}\left(t_{n-1 \ell}\right)-r_{n-1 m}-b_{0} e_{n-2}\left(t_{n-1}\right) \\
\quad-\sum_{\ell=1}^{m} b_{\ell} e_{n-1}\left(t_{n-1 \ell}\right)-\tilde{r}_{n-1}
\end{aligned}
$$

where $r_{n j}=-\int_{0}^{c_{j}} r_{n}(\tau) d \tau$ and $\tilde{r}_{n}=\int_{0}^{1} r_{n}(\tau) d \tau$. Furthermore

$$
e_{n}\left(t_{n}\right)=e_{n-1}\left(t_{n}\right)=L_{0}(1) e_{n-1}\left(t_{n-1}\right)+\sum_{\ell=1}^{m} L_{\ell}(1) e_{n-1}\left(t_{n-1 \ell}\right)+r_{n-1}(1) .
$$

These equations give in matrix notation

$$
\begin{aligned}
& \left(\begin{array}{cccc}
1 & 0 & \ldots & 0 \\
a_{10} & a_{11} & \ldots & a_{m 1} \\
\vdots & \vdots & & \vdots \\
a_{m 0} & a_{m 1} & \ldots & a_{m m}
\end{array}\right)\left(\begin{array}{c}
e_{n}\left(t_{n}\right) \\
e_{n}\left(t_{n 1}\right) \\
\vdots \\
e_{n}\left(t_{n m}\right)
\end{array}\right) \\
& =\left(\begin{array}{cccc}
L_{0}(1) & L_{1}(1) & \ldots & L_{m}(1) \\
a_{m 0}-b_{0} & a_{m 1}-b_{1} & \ldots & a_{m m}-b_{m} \\
\vdots & \vdots & \vdots \\
a_{m 0}-b_{0} & a_{m 1}-b_{1} & \ldots & a_{m m}-b_{m}
\end{array}\right)\left(\begin{array}{c}
e_{n-1}\left(t_{n-1}\right) \\
e_{n-1}\left(t_{n-11}\right) \\
\vdots \\
e_{n-1}\left(t_{n-1 m}\right)
\end{array}\right) \\
& +\left(\begin{array}{c}
r_{n 1}-r_{n-1 m}-\tilde{r}_{n-1} \\
\vdots \\
r_{n m}-r_{n-1 m}-\tilde{r}_{n-1}
\end{array}\right) .
\end{aligned}
$$

In a more compact form

$$
\widetilde{A} E_{n}=B E_{n-1}+R_{n}
$$

or

$$
E_{n}=\widetilde{A}^{-1} B E_{n-1}+\widetilde{A}^{-1} R_{n},
$$

where $E_{n}=\left(e_{n}\left(t_{n}\right), e_{n}\left(t_{n 1}\right), \ldots, e_{n}\left(t_{n m}\right)\right)^{\top}$ and

$$
\widetilde{A}=\left(\begin{array}{cc}
1 & 0 \\
a_{0} & \widehat{A}
\end{array}\right), \quad \widetilde{A}^{-1}=\left(\begin{array}{cc}
1 & 0 \\
-d & \widehat{A}^{-1}
\end{array}\right),
$$

with $d=\widehat{A}^{-1} a_{0}$. Let $\widehat{A}^{-1}=\left(\omega_{i j}\right)_{i, j=1}^{m}$. Then

$$
\widetilde{A}^{-1} B=\left(\begin{array}{ccc}
L_{0}(1) & \ldots & L_{m}(1) \\
-d_{1} L_{0}(1)+\left(a_{m 0}-b_{0}\right) \sum_{j=1}^{m} \omega_{1 j} & \ldots & -d_{1} L_{m}(1)+\left(a_{m m}-b_{m}\right) \sum_{j=1}^{m} \omega_{1 j} \\
\vdots & & \vdots \\
-d_{m} L_{0}(1)+\left(a_{m 0}-b_{0}\right) \sum_{j=1}^{m} \omega_{m j} & \ldots & -d_{m} L_{m}(1)+\left(a_{m m}-b_{m}\right) \sum_{j=1}^{m} \omega_{m j}
\end{array}\right) .
$$


Lemma 2. The matrix $\widetilde{A}^{-1} B$ given above has exactly two distinct nonzero eigenvalues $\lambda_{1}$ and $\lambda_{2}$. These eigenvalues are real and we have

$$
\begin{gathered}
\lambda_{1}+\lambda_{2}=(-1)^{m}\left(\prod_{i=1}^{m} \frac{1-c_{i}}{c_{i}}\right)\left(2+\sum_{i=1}^{m} \frac{1}{c_{i}}+\sum_{i=1}^{m} \frac{1}{1-c_{i}}\right) \\
\lambda_{1} \lambda_{2}=\left(\prod_{i=1}^{m} \frac{1-c_{i}}{c_{i}}\right)^{2} .
\end{gathered}
$$

Proof. Since $B$ is of rank 2 , the same holds for $\widetilde{A}^{-1} B$. Therefore $\widetilde{A}^{-1} B$ has exactly two nonzero eigenvalues $\lambda_{1}$ and $\lambda_{2}$. It follows from elementary linear algebra that $\lambda_{1}+\lambda_{2}$ is equal to the trace of $\widetilde{A}^{-1} B$. Then

$$
\lambda_{1}+\lambda_{2}=\operatorname{tr}\left(\widetilde{A}^{-1} B\right)=L_{0}(1)-\sum_{i=1}^{m} d_{i} L_{i}(1)+\sum_{i=1}^{m}\left(a_{m i}-b_{i}\right) \sum_{j=1}^{m} \omega_{i j} .
$$

With (2.4) and (3.7) it is now easy to verify that

$$
d_{i} L_{i}(1)=-\frac{1}{1-c_{i}} L_{0}(1)
$$

and that

$$
\sum_{i=1}^{m}\left(a_{m i}-b_{i}\right) \sum_{j=1}^{m} \omega_{i j}=1-\sum_{i=1}^{m} b_{i} \sum_{j=1}^{m} \omega_{i j}=1-\hat{b}^{\top} \widehat{A}^{-1} \mathbb{1}_{m} .
$$

Together with part d) of Lemma 1 we obtain

$$
\lambda_{1}+\lambda_{2}=\operatorname{tr}\left(\widetilde{A}^{-1} B\right)=L_{0}(1)\left(2+\sum_{i=1}^{m} \frac{1}{c_{i}}+\sum_{i=1}^{m} \frac{1}{1-c_{i}}\right) .
$$

Since the proof of (4.15) is rather technical we will not present it here; instead, we sketch the main ideas. Consider the matrix $X=\left(x_{i j}\right)_{i, j=0}^{m}$ and the corresponding characteristic polynomial $\operatorname{det}(X-\lambda I)=(-1)^{m+1} \lambda^{m+1}+\alpha_{m} \lambda^{m}+\alpha_{m-1} \lambda^{m-1}+$ $\ldots+\alpha_{0}$. Then the coefficient $\alpha_{m-1}$ is given by

$$
\alpha_{m-1}=\frac{(-1)^{m+1}}{2}\left((\operatorname{tr}(X))^{2}-\operatorname{tr}\left(X^{2}\right)\right),
$$

(see e.g. $\left[6\right.$, p. 130]). Since $\widetilde{A}^{-1} B$ is of rank 2 , the coefficient of $\lambda^{m-1}$ in the characteristic polynomial is equal to $(-1)^{m+1}$ times the product of the two nonzero eigenvalues. Applying (4.19) to $\widetilde{A}^{-1} B$, making use of (4.16), (4.17), and

$$
\begin{gathered}
\sum_{i=1}^{m}\left(a_{m i}-b_{i}\right) d_{i}=a_{m 0}-b_{0}+L_{0}(1), \\
\sum_{i=1}^{m} L_{i}(1) \sum_{k=1}^{m} \omega_{i k}=L_{0}(1)\left(1-\left(1+\sum_{i=1}^{m} \frac{1}{c_{i}}\right)\left(1+\sum_{i=1}^{m} \frac{1}{1-c_{i}}\right)\right),
\end{gathered}
$$

which one proves using parts $\mathrm{f}$ ) and e) of Lemma 1, one finally obtains

$$
\lambda_{1} \lambda_{2}=\frac{1}{2}\left(\left(\operatorname{tr}\left(\widetilde{A}^{-1} B\right)\right)^{2}-\operatorname{tr}\left(\left(\widetilde{A}^{-1} B\right)^{2}\right)\right)=\left(L_{0}(1)\right)^{2} .
$$


The eigenvalues are real since $\left(\operatorname{tr}\left(\widetilde{A}^{-1} B\right)\right)^{2}-4\left(L_{0}(1)\right)^{2}>0$ and we have

$$
\begin{aligned}
& \lambda_{1}=\frac{1}{2}\left(\operatorname{tr}\left(\widetilde{A}^{-1} B\right)+\sqrt{\left(\operatorname{tr}\left(\widetilde{A}^{-1} B\right)\right)^{2}-4\left(L_{0}(1)\right)^{2}}\right), \\
& \lambda_{2}=\frac{1}{2}\left(\operatorname{tr}\left(\widetilde{A}^{-1} B\right)-\sqrt{\left(\operatorname{tr}\left(\widetilde{A}^{-1} B\right)\right)^{2}-4\left(L_{0}(1)\right)^{2}}\right) .
\end{aligned}
$$

We can now state

Theorem 2. Let $u$ be the collocation approximation in $S_{m}^{(0)}\left(\Pi_{N}\right)$ of the solution $y$ of (1.1) and defined by (2.3)-(2.6). If $c_{m}<1$ and if the collocation parameters are symmetrical, i.e. if $c_{i}=1-c_{m+1-i}, i=1, \ldots, m$, then the collocation approximation $u$ does not converge to the solution $y$. In particular if $m=1$ there does not exist a convergent collocation method in $S_{1}^{(0)}\left(\Pi_{N}\right)$.

Proof. If $c_{m}<1$ and if the collocation parameters are symmetrical, one has $\left|L_{0}(1)\right|=1$ and

$$
\left|\lambda_{1}+\lambda_{2}\right|=2\left(1+\sum_{i=1}^{m} \frac{1}{c_{i}}\right) \geq 2(m+1) \geq 4 .
$$

Therefore the error recursion (4.13) cannot be stable since at least one eigenvalue has absolute value strictly greater than 1 .

For $m=1$, the eigenvalues of $\widetilde{A}^{-1} B$ are

$$
\begin{aligned}
& \lambda_{1}\left(c_{1}\right)=\frac{2 c_{1}^{2}-2 c_{1}-1+\sqrt{-4 c_{1}^{2}+4 c_{1}+1}}{2 c_{1}^{2}}, \\
& \lambda_{2}\left(c_{1}\right)=\frac{2 c_{1}^{2}-2 c_{1}-1-\sqrt{-4 c_{1}^{2}+4 c_{1}+1}}{2 c_{1}^{2}} .
\end{aligned}
$$

One verifies that $-1<\lambda_{1}\left(c_{1}\right)<0$ and $\lambda_{2}\left(c_{1}\right)<-1$ as $c_{1}$ varies in $(0,1)$.

Theorem 3. Assume that $g$ and $K$ in (1.1) are of class $C^{m+3}$. Let $u$ be the collocation approximation in $S_{m}^{(0)}\left(\Pi_{N}\right)$ of the solution $y$ of (1.1) and defined by (2.3)-(2.6). If $m \geq 2$ and $c_{m}<1$, then the collocation approximation $u$ converges to the solution $y$ if and only if

$$
\varrho=\max \left\{\left|\lambda_{1}\right|,\left|\lambda_{2}\right|\right\} \leq 1,
$$

where $\lambda_{1}$ and $\lambda_{2}$ are given in (4.20), (4.21) and (4.18). The collocation error $e=y-u$ satisfies

$$
\|e\|_{\infty}=\sup _{t \in I}|e(t)|=\left\{\begin{array}{ll}
\mathcal{O}\left(h^{m}\right) & \text { if } \varrho=1 \text { and } m \text { is even, } \\
\mathcal{O}\left(h^{m+1}\right) & \text { otherwise, }
\end{array} \quad h \rightarrow 0, N h=T .\right.
$$

Proof. We prove the result for constant kernel $K(t, s) \equiv 1$. For non-constant kernel we refer to Section 5 . Since $\widetilde{A}^{-1} B$ is diagonalizable, there exists a nonsingular matrix $P$ such that $\widetilde{A}^{-1} B=P D P^{-1}$ with $D=\operatorname{diag}\left(\lambda_{1}, \lambda_{2}, 0, \ldots, 0\right)$. We next multiply the recursion (4.13) by $P^{-1}$ and define $Z_{n}=P^{-1} E_{n}$. We find that

$$
Z_{n}=D Z_{n-1}+P^{-1} \widetilde{A}^{-1} R_{n}, \quad Z_{n}=D^{n} Z_{0}+\sum_{\nu=1}^{n} D^{n-\nu} P^{-1} \widetilde{A}^{-1} R_{\nu} .
$$

We conclude as in the case $c_{m}=1$. Since $\left\|E_{0}\right\|=\mathcal{O}\left(h^{m+1}\right)$ and $\left\|R_{n}\right\|=\mathcal{O}\left(h^{m+1}\right)$, 


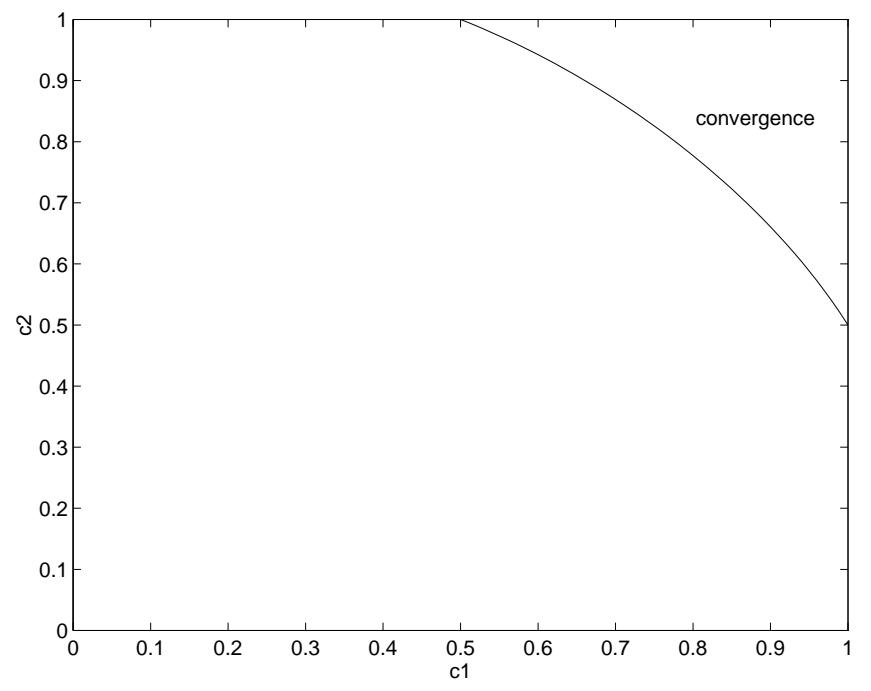

Figure 1. Domain of convergence for $m=2$

$n=1, \ldots, N,(\operatorname{see}(4.2))$, it holds that $Z_{n}=\mathcal{O}\left(h^{m}\right)$ if $\varrho=1$ and $m$ is even and $Z_{n}=\mathcal{O}\left(h^{m+1}\right)$ otherwise. With $E_{n}=P Z_{n}$, the result immediately follows.

Remarks. a) Condition $\left|L_{0}(1)\right| \leq 1$ is a necessary condition for convergence since in case of convergence $\left(L_{0}(1)\right)^{2}=\lambda_{1} \lambda_{2} \leq 1$. However it is not sufficient. This can be seen for the example $m=2, c_{1}=\frac{3}{5}, c_{2}=\frac{9}{10}$. Here $\lambda_{1}>1$ but $\left|L_{0}(1)\right|<1$.

b) Another sufficient condition for convergence is

$$
\left|\lambda_{1}+\lambda_{2}\right|=\left(\prod_{i=1}^{m} \frac{1-c_{i}}{c_{i}}\right)\left(2+\sum_{i=1}^{m} \frac{1}{c_{i}}+\sum_{i=1}^{m} \frac{1}{1-c_{i}}\right)<1 .
$$

Condition (4.23) implies (4.22), but (4.23) is stronger than (4.22). Indeed for $m=2$, $c_{1}=3 / 4$ and $c_{2}=(3+\sqrt{13}) / 8+5 \cdot 10^{-4}$, we obtain $\lambda_{1} \approx 0.9976$ and $\lambda_{2} \approx 4.929 \cdot 10^{-3}$ but $\lambda_{1}+\lambda_{2} \approx 1.0026$ (see also Method 6 of Section 6 ).

c) For a given $m$ and $c_{m}<1$, we are interested in the region in which the collocation parameters have to lie such that the collocation method is convergent. For $m=2$, we looked for the curve (in $[0,1] \times[0,1])$ defined by $\lambda_{1}\left(c_{1}, c_{2}\right)=1$. This curve is part of the ellipse given by

$$
2 c_{1}^{2}+\left(-3+2 c_{2}\right) c_{1}+1-3 c_{2}+2 c_{2}^{2}=0 .
$$

This ellipse has center $(1 / 2,1 / 2)$, principal axes $(1,-1)$ and $(1,1)$ and half axes $\sqrt{2} / 2$ and $\sqrt{6} / 6$. One has convergence if $c_{1}$ and $c_{2}$ lie in $(0,1) \times(0,1)$ and "above" this curve (see Figure 1).

d) A necessary and sufficient condition similar to (4.22) for convergence of piecewise polynomial spline approximations with possible jump discontinuities for solving (1.1) with $K(t, t)=0, t \in I, g(0)=g^{\prime}(0)=0$, has been given in [5].

\section{Convergence for general Kernels}

In this section we prove the results of Theorems 1 and 3 for general, non-constant kernels. We first rewrite (4.4) for $n-1$ with $j=m$ and subtract it from (4.4). We 
obtain

$$
\begin{array}{rl}
\int_{0}^{c_{j}} K & K\left(t_{n j}, t_{n}+\tau h\right) e_{n}\left(t_{n}+\tau h\right) d \tau \\
= & \int_{0}^{c_{m}} K\left(t_{n-1 m}, t_{n-1}+\tau h\right) e_{n-1}\left(t_{n-1}+\tau h\right) d \tau \\
& -\int_{0}^{1} K\left(t_{n j}, t_{n-1}+\tau h\right) e_{n-1}\left(t_{n-1}+\tau h\right) d \tau \\
& +\sum_{\nu=0}^{n-2} \int_{0}^{1}\left(K\left(t_{n-1 m}, t_{\nu}+\tau h\right)-K\left(t_{n j}, t_{\nu}+\tau h\right)\right) e_{\nu}\left(t_{\nu}+\tau h\right) d \tau
\end{array}
$$

We again have to distinguish between the two cases $c_{m}=1$ and $c_{m}<1$. We investigate in detail the case $c_{m}<1$. The "easier" case $c_{m}=1$ can be treated quite similarly. We next rewrite (5.1) with $n$ replaced by $n-1$ and subtract it from (5.1). This yields

$$
\begin{array}{rl}
\int_{0}^{c_{j}} & K\left(t_{n j}, t_{n}+\tau h\right) e_{n}\left(t_{n}+\tau h\right) d \tau=\int_{0}^{c_{j}} K\left(t_{n-1 j}, t_{n-1}+\tau h\right) e_{n-1}\left(t_{n-1}+\tau h\right) d \tau \\
& +\int_{0}^{c_{m}} K\left(t_{n-1 m}, t_{n-1}+\tau h\right) e_{n-1}\left(t_{n-1}+\tau h\right) d \tau \\
& -\int_{0}^{1} K\left(t_{n j}, t_{n-1}+\tau h\right) e_{n-1}\left(t_{n-1}+\tau h\right) d \tau \\
& -\int_{0}^{c_{m}} K\left(t_{n-2 m}, t_{n-2}+\tau h\right) e_{n-2}\left(t_{n-2}+\tau h\right) d \tau \\
& +\int_{0}^{1} K\left(t_{n-1 j}, t_{n-2}+\tau h\right) e_{n-2}\left(t_{n-2}+\tau h\right) d \tau \\
& +\int_{0}^{1}\left(K\left(t_{n-1 m}, t_{n-2}+\tau h\right)-K\left(t_{n j}, t_{n-2}+\tau h\right)\right) e_{n-2}\left(t_{n-2}+\tau h\right) d \tau \\
& +\sum_{\nu=0}^{n-3} \int_{0}^{1}\left(K\left(t_{n-1 m}, t_{\nu}+\tau h\right)-K\left(t_{n j}, t_{\nu}+\tau h\right)\right. \\
&
\end{array}
$$

We now use

$$
\begin{aligned}
& K\left(t_{n-1 m}, t_{n-2}+\tau h\right)-K\left(t_{n j}, t_{n-2}+\tau h\right)=h\left(c_{m}-c_{j}-1\right) K_{t}\left(\xi_{n}, t_{n-2}+\tau h\right), \\
& K\left(t_{n-1 m}, t_{\nu}+\tau h\right)-K\left(t_{n j}, t_{\nu}+\tau h\right)-K\left(t_{n-2 m}, t_{\nu}+\tau h\right)+K\left(t_{n-1 j}, t_{\nu}+\tau h\right) \\
& \quad=h^{2} \alpha_{m j} K_{t t}\left(\eta_{n}, t_{\nu}+\tau h\right),
\end{aligned}
$$

where $t_{n-1 m}<\xi_{n}<t_{n j}, t_{n-2 m}<\eta_{n}<t_{n j}$ and the $\alpha_{m j}$ are constants depending only on the parameters $c_{j}$ and insert (4.3) in the above equation. According to (3.1) we may write the integrals of the kernel multiplied by a Lagrange polynomial as

$$
\int_{0}^{c_{j}} K\left(t_{n j}, t_{n}+\tau h\right) L_{\ell}(\tau) d \tau=K\left(t_{n}, t_{n}\right) a_{j \ell}+\mathcal{O}(h), \quad \ell=0, \ldots, m
$$


and

$$
\int_{0}^{1} K\left(t_{n j}, t_{n-1}+\tau h\right) L_{\ell}(\tau) d \tau=K\left(t_{n-1}, t_{n-1}\right) b_{\ell}+\mathcal{O}(h), \quad \ell=0, \ldots, m,
$$

etc. and divide by $K\left(t_{n}, t_{n}\right)$ which is possible because we assumed that $|K(t, t)| \geq$ $\kappa>0$. For $j=1, \ldots, m$, we obtain

$$
\begin{aligned}
\left(a_{j 0}\right. & +\mathcal{O}(h)) e_{n}\left(t_{n}\right)+\sum_{\ell=1}^{m}\left(a_{j \ell}+\mathcal{O}(h)\right) e_{n}\left(t_{n \ell}\right) \\
& =\left(a_{j 0}+\mathcal{O}(h)\right) e_{n-1}\left(t_{n-1}\right)+\sum_{\ell=1}^{m}\left(a_{j \ell}+\mathcal{O}(h)\right) e_{n-1}\left(t_{n-1 \ell}\right) \\
& +\left(a_{m 0}-b_{0}+\mathcal{O}(h)\right) e_{n-1}\left(t_{n-1}\right)+\sum_{\ell=1}^{m}\left(a_{m \ell}-b_{\ell}+\mathcal{O}(h)\right) e_{n-1}\left(t_{n-1 \ell}\right) \\
& -\left(a_{m 0}-b_{0}+\mathcal{O}(h)\right) e_{n-2}\left(t_{n-2}\right)-\sum_{\ell=1}^{m}\left(a_{m \ell}-b_{\ell}+\mathcal{O}(h)\right) e_{n-2}\left(t_{n-2 \ell}\right) \\
& +h^{2} \sum_{\nu=0}^{n-3} C_{j}^{(n \nu)} e_{\nu}\left(t_{\nu}\right)+h^{2} \sum_{\nu=0}^{n-3} \sum_{\ell=1}^{m} D_{j \ell}^{(n \nu)} e_{\nu}\left(t_{\nu \ell}\right)+r_{n j},
\end{aligned}
$$

where $r_{n j}$ contains all the remainder terms and satisfies $r_{n j}=\mathcal{O}\left(h^{m+2}\right)$ since $r_{n}(\tau)-r_{n-1}(\tau)=\mathcal{O}\left(h^{m+2}\right)$ (see (4.2)). Next we rewrite (4.12) for $n-1$ and subtract it from (4.12). Using the same notations as in Section 4, Case II, we finally obtain the error recursion for $E_{n}=\left(e_{n}\left(t_{n}\right), e_{n}\left(t_{n 1}\right), \ldots, e_{n}\left(t_{n m}\right)\right)^{\top}$,

$$
(\widetilde{A}+\mathcal{O}(h)) E_{n}=(\widetilde{A}+B+\mathcal{O}(h)) E_{n-1}-(B+\mathcal{O}(h)) E_{n-2}+h^{2} \sum_{\nu=0}^{n-3} D_{n \nu} E_{\nu}+R_{n}^{\prime},
$$

with $R_{n}^{\prime}=\mathcal{O}\left(h^{m+2}\right)$ and with bounded matrices $D_{n \nu}$ (whose meaning is clear from the above). If $h$ is sufficiently small, the matrix $\widetilde{A}+\mathcal{O}(h)$ is invertible and it follows that

$$
E_{n}=\left(I+\widetilde{A}^{-1} B+h V_{n-1}\right) E_{n-1}-\left(\widetilde{A}^{-1} B+h W_{n-2}\right) E_{n-2}+h^{2} \sum_{\nu=0}^{n-3} G_{n \nu} E_{\nu}+R_{n}^{\prime \prime},
$$

where $G_{n \nu}, V_{n}$ and $W_{n}$ are bounded matrices and $R_{n}^{\prime \prime}=\mathcal{O}\left(h^{m+2}\right)$. We now define

$$
\begin{gathered}
X_{n}=\left(\begin{array}{c}
E_{n} \\
E_{n-1}
\end{array}\right), \quad X_{0}=\left(\begin{array}{c}
E_{0} \\
0
\end{array}\right), \quad F=\left(\begin{array}{cc}
I+\widetilde{A}^{-1} B & -\widetilde{A}^{-1} B \\
I & 0
\end{array}\right), \\
H_{n-1}=\left(\begin{array}{cc}
V_{n-1} & -W_{n-2} \\
0 & 0
\end{array}\right), \quad M_{n \nu}=\left(\begin{array}{cc}
G_{n \nu} & 0 \\
0 & 0
\end{array}\right), \quad R_{n}=\left(\begin{array}{c}
R_{n}^{\prime \prime} \\
0
\end{array}\right)
\end{gathered}
$$

and obtain

$$
X_{n}=\left(F+h H_{n-1}\right) X_{n-1}+h^{2} \sum_{\nu=0}^{n-3} M_{n \nu} X_{\nu}+R_{n} .
$$

If $-1 \leq \lambda_{1}, \lambda_{2}<1$, the matrix $F$ is diagonalizable and there exists a nonsingular matrix $T$ such that

$$
T^{-1} F T=D=\operatorname{diag}\left(1, \ldots, 1, \lambda_{1}, \lambda_{2}, 0, \ldots, 0\right),
$$


with each diagonal block $\operatorname{diag}(1, \ldots, 1)$ and $\operatorname{diag}\left(\lambda_{1}, \lambda_{2}, 0, \ldots, 0\right)$ of dimension $m+$ 1. Multiply (5.2) by $T^{-1}$ and define $Z_{n}=T^{-1} X_{n}$ to obtain

$$
Z_{n}=\left(D+h T^{-1} H_{n-1} T\right) Z_{n-1}+h^{2} \sum_{\nu=0}^{n-3} T^{-1} M_{n \nu} T Z_{\nu}+T^{-1} R_{n} .
$$

Taking norms, we arrive at an inequality of the form

$$
\left\|Z_{n}\right\| \leq(1+\mathcal{O}(h))\left\|Z_{n-1}\right\|+h^{2} L \sum_{\nu=0}^{n-3}\left\|Z_{\nu}\right\|+\mathcal{O}\left(h^{m+2}\right) .
$$

Lemma 6 of [7] shows that $\left\|Z_{n}\right\|=\mathcal{O}\left(h^{m+1}\right)$ and the result of Theorem 3 immediately follows.

It remains to show convergence of order $m$ if $\lambda_{1}=1$. This can be done in the following way. We first write the collocation approximation $u_{n}$ on $\left[t_{n}, t_{n+1}\right]$ in the form

$$
u_{n}\left(t_{n}+\tau h\right)=\sum_{\ell=1}^{m} \bar{L}_{\ell}(\tau) u_{n}\left(t_{n \ell}\right)+h^{m} \frac{u_{n}^{(m)}\left(t_{n}^{+}\right)}{m !} \prod_{i=1}^{m}\left(\tau-c_{i}\right), \quad \tau \in[0,1],
$$

where $\bar{L}_{\ell}(\tau), \ell=1, \ldots, m$, are the Lagrange polynomials of degree $m-1$ defined with respect to the points $0<c_{1}<\ldots<c_{m}<1$, i.e.

$$
\bar{L}_{\ell}(\tau)=\prod_{\substack{k=1 \\ k \neq \ell}}^{m}\left(\tau-c_{k}\right) /\left(c_{\ell}-c_{k}\right), \quad \ell=1, \ldots, m
$$

The exact solution can be written in a similar way, namely

$$
y\left(t_{n}+\tau h\right)=\sum_{\ell=1}^{m} \bar{L}_{\ell}(\tau) y\left(t_{n \ell}\right)+h^{m} \frac{y^{(m)}\left(\zeta_{n}(\tau)\right)}{m !} \prod_{i=1}^{m}\left(\tau-c_{i}\right), \quad \tau \in[0,1],
$$

such that the error on $\left[t_{n}, t_{n+1}\right]$ can be expressed as

$$
e_{n}\left(t_{n}+\tau h\right)=\sum_{\ell=1}^{m} \bar{L}_{\ell}(\tau) e_{n}\left(t_{n \ell}\right)+\mathcal{O}\left(h^{m}\right), \quad \tau \in[0,1] .
$$

We now proceed as in the convergence proof of collocation approximations in the spline space $S_{m-1}^{(-1)}\left(\Pi_{N}\right)$. Let $\bar{A}=\left(\bar{a}_{j \ell}\right)_{j, \ell=1}^{m}$ and $\bar{b}=\left(\bar{b}_{1}, \ldots, \bar{b}_{m}\right)^{\top}$ be the coefficients of the implicit Runge-Kutta method defined by

$$
\bar{a}_{j \ell}=\int_{0}^{c_{j}} \bar{L}_{\ell}(\tau) d \tau, \quad \bar{b}_{\ell}=\int_{0}^{1} \bar{L}_{\ell}(\tau) d \tau, \quad j, \ell=1, \ldots, m .
$$

Since this Runge-Kutta method satisfies condition $C(m)$ (see Theorem II.7.8 of [8]), the matrix $\bar{A}$ is invertible. We insert (5.3) into (5.1) and proceed as previously. We obtain the following recursion for $E_{n}=\left(e_{n}\left(t_{n 1}\right), \ldots, e_{n}\left(t_{n m}\right)\right)^{\top}$ :

$$
(\bar{A}+\mathcal{O}(h)) E_{n}=\left(\mathbb{1}_{m} e_{m}^{\top} \bar{A}-\mathbb{1}_{m} \bar{b}^{\top}+\mathcal{O}(h)\right) E_{n-1}+h \sum_{\nu=0}^{n-2} Q_{n \nu} E_{\nu}+\mathcal{O}\left(h^{m}\right) .
$$


If $h$ is sufficiently small, $\bar{A}+\mathcal{O}(h)$ is invertible and it follows that

$$
E_{n}=M_{0} E_{n-1}+h \sum_{\nu=0}^{n-2} \widetilde{Q}_{n \nu} E_{\nu}+\mathcal{O}\left(h^{m}\right),
$$

where

$$
M_{0}=\bar{A}^{-1} \mathbb{1}_{m}\left(e_{m}^{\top} \bar{A}-\bar{b}^{\top}\right)
$$

and $e_{m}=(0, \ldots, 0,1)^{\top} \in \mathbb{R}^{m}$.

Lemma 3. Let $M_{0}$ be defined as above. Then $M_{0}$ has rank one and its only nonzero eigenvalue is

$$
\bar{R}(\infty)=(-1)^{m} \prod_{i=1}^{m} \frac{1-c_{i}}{c_{i}}
$$

where $\bar{R}(z)$ denotes the stability function of the Runge-Kutta method $(\bar{A}, \bar{b}, \hat{c})$.

Proof. $M_{0}$ has rank one because $\mathbb{1}_{m}\left(e_{m}^{\top} \bar{A}-\bar{b}^{\top}\right)$ has rank one. Therefore the nonzero eigenvalue of $M_{0}$ is equal to its trace. Let $\bar{A}^{-1}=\left(\bar{\omega}_{i j}\right)_{i, j=1}^{m}$. Then

$$
\operatorname{tr}\left(M_{0}\right)=\sum_{i=1}^{m}\left(\bar{a}_{m i}-\bar{b}_{i}\right) \sum_{j=1}^{m} \bar{\omega}_{i j}=1-\bar{b}^{\top} \bar{A}^{-1} \mathbb{1}_{m}=\bar{R}(\infty) .
$$

The result now follows from Theorem IV.3.9 of [9].

Since $M_{0}$ is diagonalizable, there exists a nonsingular matrix $P$ such that $M_{0}=$ $P D P^{-1}$ with $D=\operatorname{diag}(\bar{R}(\infty), 0, \ldots, 0)$. We now multiply (5.4) by $P^{-1}$ and define $Z_{n}=P^{-1} E_{n}$. Hence

$$
Z_{n}=D Z_{n-1}+h \sum_{\nu=0}^{n-2} P^{-1} Q_{n \nu}^{\prime} P Z_{\nu}+\mathcal{O}\left(h^{m}\right) .
$$

Since $\lambda_{1}=1$, by Lemma 3 and (4.15) it now holds that

$$
\|D\|=|\bar{R}(\infty)|=\prod_{i=1}^{m} \frac{1-c_{i}}{c_{i}}=\sqrt{\lambda_{2}}<1 .
$$

Therefore

$$
\left\|Z_{n}\right\| \leq \sqrt{\lambda_{2}}\left\|Z_{n-1}\right\|+h L \sum_{\nu=0}^{n-2}\left\|Z_{\nu}\right\|+\mathcal{O}\left(h^{m}\right)
$$

and applying Lemma 6 of [7] we conclude that $\left\|Z_{n}\right\|=\mathcal{O}\left(h^{m}\right)$. Thus $\left\|E_{n}\right\|=\mathcal{O}\left(h^{m}\right)$ and the result follows from (5.3). In the case $\lambda_{1}=1$ the estimate $\|e\|_{\infty}=\mathcal{O}\left(h^{m}\right)$ is optimal, the order of convergence does not exceed $m$. This has been confirmed in numerous numerical experiments.

\section{Discretized COLLOCATION AND NUMERICAL ILLUSTRATION}

The integrals in (2.5) can in general not be computed explicitly, and thus one has to use appropriate quadrature formulas to approximate them. Since the collocation methods are convergent of order $m+1$ (see Theorems 1 and 3), one has to choose quadrature formulas of order at least $m+1$ (i.e. of degree of precision at least 
$m)$. It is natural to use the quadrature formula based on $b=\left(b_{0}, \ldots, b_{m}\right)^{\top}$ and $c=\left(c_{0}, \ldots, c_{m}\right)^{\top}$ (i.e. satisfying $\left.B(m+1)\right)$ to approximate the definite integral of a function over the interval $[0,1]$,

$$
\int_{0}^{1} f(\tau) d \tau \approx \sum_{j=0}^{m} b_{j} f\left(c_{j}\right)
$$

To approximate the definite integral of a function over the interval $\left[0, c_{j}\right]$, one has two possibilities. If the kernel $K(t, s)$ is defined also for $s \geq t$, one can use

$$
\int_{0}^{c_{j}} f(\tau) d \tau \approx \sum_{\ell=0}^{m} a_{j \ell} f\left(c_{\ell}\right) .
$$

This method uses $\left(a_{j \ell}\right)_{j, \ell=0}^{m}$ defined by $C(m+1)$. If $K(t, s)$ is only defined for $s \leq t$ or if one wants to use only values of $K(t, s)$ with $s \leq t$, one uses

$$
\int_{0}^{c_{j}} f(\tau) d \tau \approx c_{j} \sum_{\ell=0}^{m} b_{\ell} f\left(c_{j} c_{\ell}\right)
$$

Let $\hat{u} \in S_{m}^{(0)}\left(\Pi_{N}\right)$ denote the resulting discretized continuous collocation approximation of $y$. One shows that the error $\hat{e}=y-\hat{u}$ has the same order of magnitude as $e=y-u$. It is sufficient to show that $u-\hat{u}$ satisfies the estimate

$$
\|u-\hat{u}\|_{\infty}=\mathcal{O}\left(h^{m+1}\right) .
$$

This again leads to recursions of the form encountered in Sections 4 and 5. We omit the details. The convergence analysis of the fully discretized method in the case $c_{m}=1$ can be found in [11].

To illustrate the theoretical findings of the preceding sections, we solved (1.1) on $I=[0,1]$ with $K(t, s)=\exp (-t s)$ and $g(t)$ such that $y(t)=\exp (-t) \cos (t)$. We used the quadrature formulas (6.1) and (6.2) to approximate the integrals in (2.5). In Figure 2 we represented the maximal error on $[0,1]$ versus the stepsize $h=1 / N$ with $N=2,4,8, \ldots, 256$. We used double logarithmic scales such that one observes the slope $p$ for a method whenever this method has order $p$. We employed the following methods, those with $c_{m}=1$ are represented in Figure 2.a), those with $c_{m}<1$ in Figure 2.b).

Method 1: $m=1, c_{1}=1$, (o in Fig. 2.a)).

Method 2: $m=2, c_{1}=1 / 2, c_{2}=1,(\times$ in Fig. 2.a $)$ ).

Method 3: $m=2, c_{1}=2 / 3, c_{2}=1,(+$ in Fig. 2.a)).

Method 4: $m=3, c_{1}=1 / 3, c_{2}=2 / 3, c_{3}=1$, (* in Fig. 2.a)).

Method 5: $m=2, c_{1}=4 / 5, c_{2}=19 / 20$, (o in Fig. 2.b)).

Method 6: $m=2, c_{1}=3 / 4, c_{2}=(3+\sqrt{13}) / 8,(\times$ in Fig. 2.b $\left.)\right)$.

Method 7: $m=3, c_{1}=3 / 7, c_{2}=4 / 5, c_{3}=8 / 9,(+$ in Fig. 2.b)).

Method 8: $m=4, c_{1}=4 / 10, c_{2}=7 / 10, c_{3}=8 / 10, c_{4}=9 / 10,(*$ in Fig. 2.b)).

It can be seen that methods $1,3,4,5,7$ and 8 converge with order $m+1$. For methods 2 and 6 one observes an order reduction: these methods only converge with order $m$. For method $2, R(\infty)=1$, for method $6,0<\lambda_{2}<\lambda_{1}=1$. 

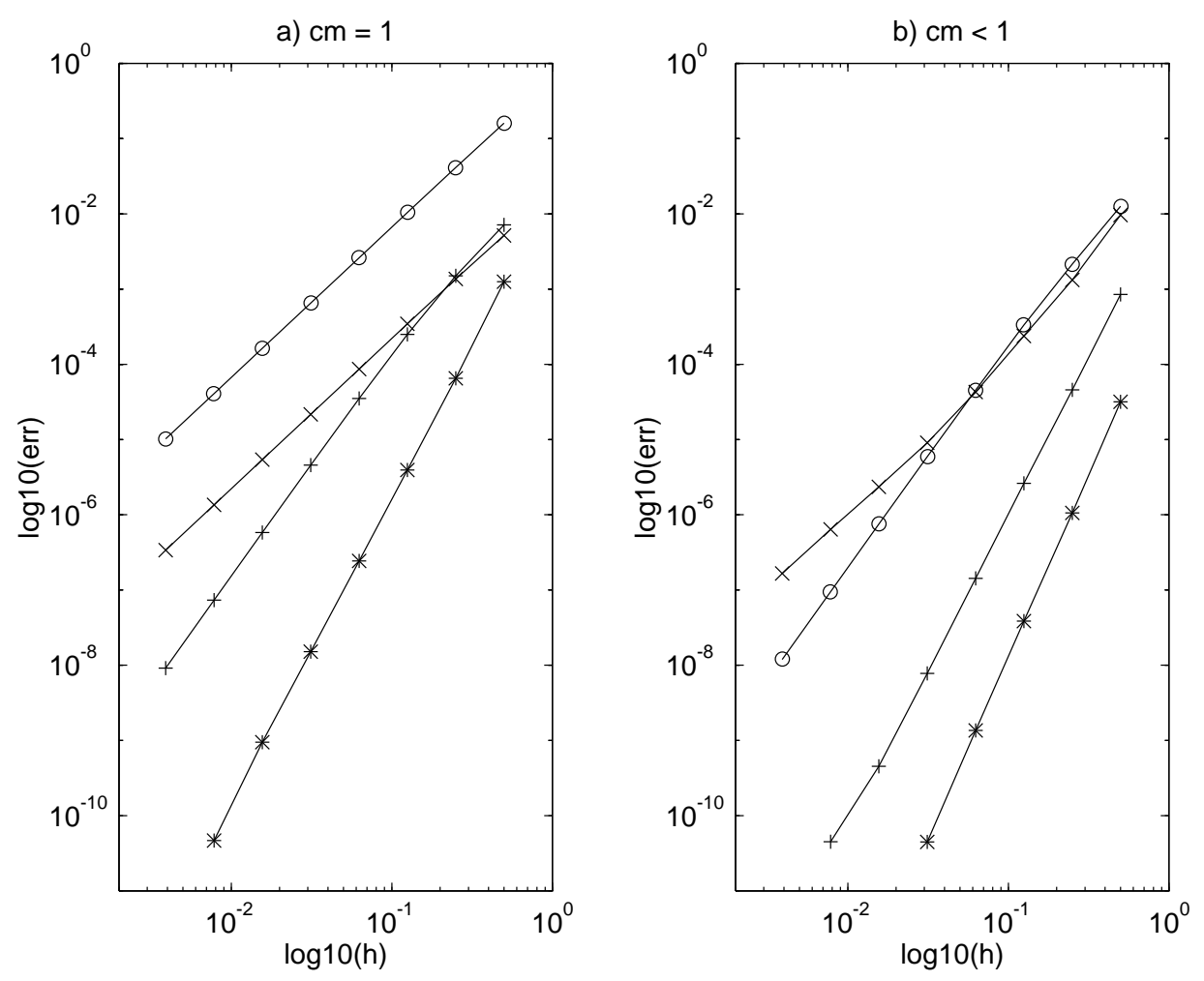

FiguRE 2. Error versus stepsize

\section{Concluding Remarks}

1) For collocation approximations in $S_{m}^{(0)}\left(\Pi_{N}\right)$ of the solution of a first kind Volterra equation, one does in general not observe (local) superconvergence, neither at the mesh points $t_{n}$ nor at the collocation points $t_{n j}$. The error is $\mathcal{O}\left(h^{m+1}\right)$ in all points of $[0, T]$ if $-1 \leq R(\infty)<1$ or $-1 \leq \lambda_{1}, \lambda_{2}<1$ and $\mathcal{O}\left(h^{m}\right)$ if $m$ is even and $\varrho=1$ (with $c_{m}<1$ ) or $R(\infty)=1$ (with $c_{m}=1$ ). However in the latter case superconvergence is possible if one chooses as collocation parameters $0<c_{1}<$ $\ldots<c_{m}=1$ the $m$ nonzero Lobatto points in $(0,1]$ and evaluates the collocation approximation at the points $t_{n}+v_{j} h$ where $\left\{v_{j}: j=1, \ldots, m\right\}$ are the Gauss points in $(0,1)$. In this case one observes numerically $\left|e_{n}\left(t_{n}+v_{j} h\right)\right|=\mathcal{O}\left(h^{m+1}\right)$, $j=1, \ldots, m, n=0, \ldots, N-1$. This is the case for example if $c_{1}=1 / 2, c_{2}=1$ and $v_{1}=(3-\sqrt{3}) / 6, v_{2}=(3+\sqrt{3}) / 6$. This property will be investigated elsewhere. It is similar to the increase of the order of convergence for collocation approximations in $S_{m}^{(-1)}\left(\Pi_{N}\right)$ established in [3].

2) The results of this paper can be extended to nonlinear first kind Volterra equations

$$
\int_{0}^{t} k(t, s, y(s)) d s=g(t), \quad t \in[0, T]
$$


Here, in addition to the conditions for $g$ (cf. Section 1), one has to assume that $k$ is sufficiently smooth and $\partial k / \partial y(t, t, z)$ is bounded away from zero for all $t \in[0, T]$ and $z$ in a neighbourhood of the solution. In the error recursion (4.4) $K\left(t_{n j}, t_{n}+\tau h\right)$ then has to be replaced by $\partial k / \partial y\left(t_{n j}, t_{n}+\tau h, \cdot\right)$ where $\cdot$ is between $y\left(t_{n}+\tau h\right)$ and $u\left(t_{n}+\tau h\right)$.

\section{ACKNOWLEDGEMENT}

We thank Professor Ernst Hairer (Université de Genève) for suggesting the proof of part d) of Lemma 1.

\section{REFERENCES}

1. H. Brunner, Discretization of Volterra integral equations of the first kind, Math. Comp., 31 (1977), 708-716. MR 56:10076

2. H. Brunner, Discretization of Volterra integral equations of the first kind (II), Numer. Math., 30 (1978), 117-136. MR 58:3578

3. H. Brunner, Superconvergence of collocation methods for Volterra integral equations of the first kind, Computing, 21 (1979), 151-157. MR 83a:65125

4. H. Brunner and P.J. van der Houwen, The Numerical Solution of Volterra Equations, NorthHolland, Amsterdam, 1986. MR 88g:65136

5. P.P.B. Eggermont, Collocation for Volterra integral equations of the first kind with iterated kernel, SIAM J. Numer. Anal., 20 (1983), 1032-1048. MR 85i:65170

6. W. Greub, Linear Algebra, Fourth Edition, Springer-Verlag, New York Heidelberg Berlin, 1975. MR 51:5615

7. E. Hairer, Ch. Lubich and S.P. Nørsett, Order of convergence of one-step methods for Volterra integral equations of the second kind, SIAM J. Numer. Anal., 20 (1983), 569-579. MR 84g:65163

8. E. Hairer, S.P. Nørsett and G. Wanner, Solving Ordinary Differential Equations I. Nonstiff Problems, Second Revised Edition, Springer-Verlag, Berlin Heidelberg, 1993. MR 94c:65005

9. E. Hairer and G. Wanner, Solving Ordinary Differential Equations II. Stiff and DifferentialAlgebraic Problems, Springer-Verlag, Berlin Heidelberg, 1991. MR 92a:65016

10. F. de Hoog and R. Weiss, On the solution of Volterra integral equations of the first kind, Numer. Math., 21 (1973), 22-32. MR 51:7335

11. F. de Hoog and R. Weiss, High order methods for Volterra integral equations of the first kind, SIAM J. Numer. Anal., 10 (1973), 647-664. MR 51:9554

12. H.S. Hung, The numerical solution of differential and integral equations by spline functions, MRC Tech. Summary Rep. 1053, Mathematics Research Center, University of Wisconsin, Madison, 1970.

Institut de Mathématiques, Université de Fribourg, CH-1700 Fribourg, Switzerland

E-mail address: jean-paul.kauthen@unifr.ch, kauthen@bluewin.ch

Department of Mathematics and Statistics, Memorial University of Newfoundland, St. John's, Newfoundland, Canada A1C 5S7

E-mail address: hbrunner@morgan.ucs.mun.ca 